\title{
Mesiodens: la influencia en la autoestima del niño. Reporte de caso.
}

\section{Resumen}

Se denomina diente supernumerario todo y cualquier elemento que se presente en número mayor de lo considerado normal en un arco dental. Cuando se localiza entre incisivos centrales y de formato cónica, se denomina mesiodens pudiendo causar importantes alteraciones comportamentales y psicológicas en su portador. El presente artículo relata el caso de un niño de 9 años de edad, portador de mesiodiens, con queja principal de que su boca era de "tiburón" y que afectaba su autoestima. Se realizó adecuación del
Laís Cecilio da Costa, ${ }^{1}$ Natália da Silva Mota, ${ }^{1}$ Jose Massao Miasato, ${ }^{2}$ Leila Chevitarese, ${ }^{2}$ Luciana Alves Herdy da Silva. ${ }^{2}$ medio bucal y exodoncia del elemento, con retorno 3 meses después para acompañamiento. Se observaron cambios en su comportamiento y convivencia social después de la remoción del mesiodens. Este relato buscó enfatizar las alteraciones conductuales y psicológicas que pueden ocurrir en niños con anomalías dentales en la región anterior y cómo el tratamiento posibilita cambios positivos, presentando mejoría en su autoestima e interacciones sociales.

Palabras clave: Diente supernumerario, diagnóstico, autoimagen. 


\section{Mesiodente: a influência na autoestima da criança. Relato de caso.}

\section{Resumo}

É denominado dente supranumerário todo e qualquer elemento que se apresente em número maior do que o considerado normal em uma arcada. Quando localizado entre incisivos centrais e de formato conóide, é denominado mesiodente, podendo causar importantes alterações comportamentais e psicológicas em seu portador. O presente artigo relata o caso de uma criança de 9 anos de idade e portadora de mesiodente com queixa principal de que sua boca era de "tubarão" e que afetava sua autoestima. Foi realizada adequação do meio bucal e exodontia doelemento, com retorno 3 meses depois para acompanhamento. Observouse mudanças em seu comportamento e convívio social após a remoção do mesiodente. Esse relato buscou enfatizar as alterações comportamentais e psicológicas que podem ocorrer em crianças com anomalias dentárias na região anterior e como o tratamento possibilita mudanças positivas, apresentando melhora em sua autoestima e interações sociais.

Palavras chave: Dente supranumerário, diagnóstico, autoestima.

Case report

\section{Mesiodens: the influence on the child's self-esteem. Clinical case report.}

\begin{abstract}
A supernumerary tooth any dental element that presents itself in greater number than considered normal for the maxilla or mandible. When located between the maxillary central incisors, it is called mesiodens and may cause important behavioral and psychological changes in the patient. This report presents case of a 9 yearold male patient who had a supernumerary tooth and complained that he had "shark's teeth" which affected his self-esteem. Adequacy of the buccal environment and extraction of the supernumerary tooth
\end{abstract}

were performed. The patient returned 3 months later for follow-up. Changes in behavior and social interaction after the removal of the mesiodens were observed. This report aimed to emphasize the behavioral and psychological changes that may occur in children with previous dental anomalies in the maxillary incisor region and how early treatment will bring aesthetic, psychological and social benefits to them

Key words: Supernumerary tooth, diagnosis, self-esteem. 


\section{Introducción}

Los dientes supernumerarios conforman un tipo de anomalía caracterizada por la presencia de dientes más allá del número esperado o considerado normal en una arcada o hemiarcada. Pueden erupcionar en el tiempo previsto, impactarse, invertirse $\mathrm{o}$ incluso presentarse en posición ectópica. Pueden ocurrir de forma aislada o múltiple, pudiendo aparecer uni o bilateralmente tanto en la mandíbula como en el maxilar. ${ }^{1-13}$ Cualquier diente que esté por encima del número normal en las denticiones primarias y permanentes se considera un supernumerario. $^{2}$

Los dientes supernumerarios se presentan tanto en el maxilar como en la mandíbula, teniendo mayor prevalencia en la región anterior del maxilar y en pacientes con síndromes o patologías sistémicas. Su origen todavía es poco conocido y está asociado a diversas teorías. Algunos autores creen que pueden estar asociados al hiperdesarrollo del germen dentario, otros al mal desarrollo del germen en el momento de su evolución, o incluso a la teoría del atavismo, o sea, la reaparición de una condición ancestral. ${ }^{3,7}$

En lo que se refiere al tipo, el más común es el mesiodens, considerado un elemento supernumerario que se ubica en la región de la línea media del paciente, aislado o en pares, erupcionado, incluido o invertido. Normalmente se presenta como un diente pequeño, con forma cónica, de raíz corta 4 y dismorfo. ${ }^{5}$ Los mesiodens representan, en promedio, el $80 \%$ de las ocurrencias de dientes supernumerarios. ${ }^{3}$

El presente estudio tiene como objetivo relatar a través de un caso clínico, los aspectos psicológicos y clínicos en un niño portador de mesiodens.

\section{Reporte de caso}

Paciente de sexo masculino de 8 años de edad, se presentó en la clínica integrada del niño y adolescente del curso de odontología de la Universidad Unigranrio - Duque de Caxias, Río de Janeiro, en octubre de 2017, con la queja de que su boca era de "tiburón" (SIC). El mismo relató tener vergüenza de sonreír y sentirse excluido entre los amigos de la escuela, caracterizando una situación de "bullying", además de mostrar retraimiento cuando hablaba con las personas fuera de su convivencia diaria. Después del relato del paciente, se llenó la ficha clínica en la que el padre relató que, desde la erupción de todos los dientes anteriores, también había aparecido un diente entre los "dientes delanteros" (SIC). En el examen clínico, se identificó un elemento de forma amorfa entre los incisivos centrales superiores que podría tratarse de un mesiodens (Figuras $1 \mathrm{y}$ 2 ), lesiones cariosas en las superficies

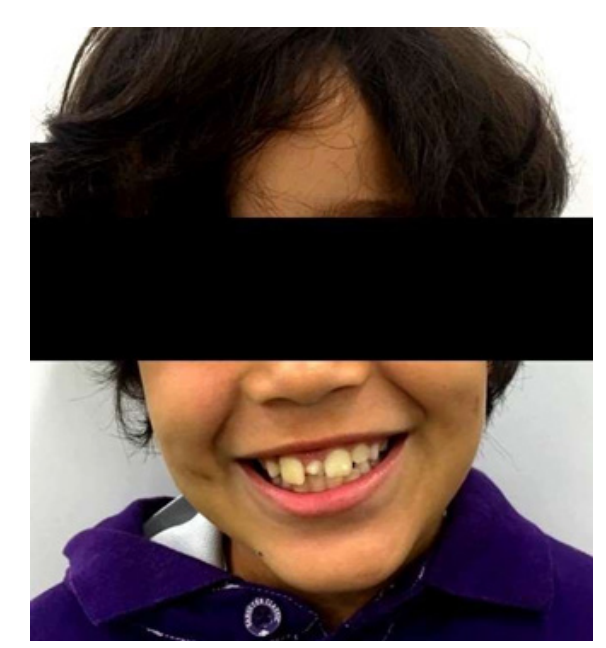

Figura 1. Paciente presentando mesiodens al sonreír. 


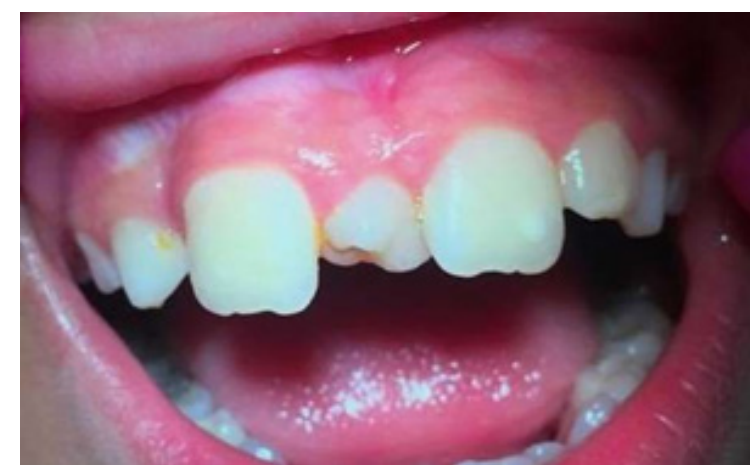

Figura 2. Visión Intraoral.

vestibular y oclusal del elemento 75 y en proximal del elemento 84. De este modo, se le instruyó la higiene oral al paciente y se le solicitó la toma de una radiografía panorámica (Figura3) para la visualización de los elementos en formación, buscando una mejor planificación del caso. Con interés en publicar el trabajo, se le pidió al responsable del niño, la firma para el término de consentimiento de uso de imágenes, como se suele pedir antes de iniciar el tratamiento en la clínica.

En las siguientes consultas, se realizaron restauraciones masivas, con remoción de tejido cariado con fresa esférica laminada (de fisuras) y cucharita de dentina en los molares primarios 75 y 84, utilizando cemento de ionómero de vidrio (CIV)

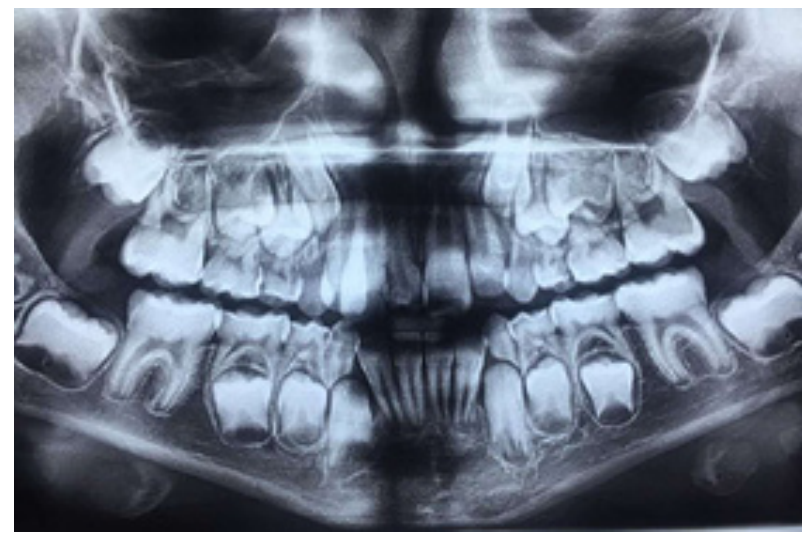

Figura 3. Radiografia panorámica con diente supernumerario. y resina compuesta como materiales restauradores. En la cuarta consulta, se preparó el paciente para exodoncia, ${ }^{11}$ con explicaciones sencillas sobre los signos que él presentaría, debido a su primer contacto con la anestesia. El paciente oscilaba entre levemente positivo y levemente negativo según la clasificación de Frankl. Se utilizó anestésico tópico y técnica infiltrativa en la región de incisivos y fosa canina en ambos lados, con desvío para incisivos. Para la remoción del elemento dentario, se utilizó un fórceps infantil. A continuación, se realizó el taponamiento con gasa para el control del sangramiento (Figuras $4 \mathrm{y}$ 5).

Después de la cirugía, se prescribió paracetamol de $500 \mathrm{mg}$ en caso de dolor.

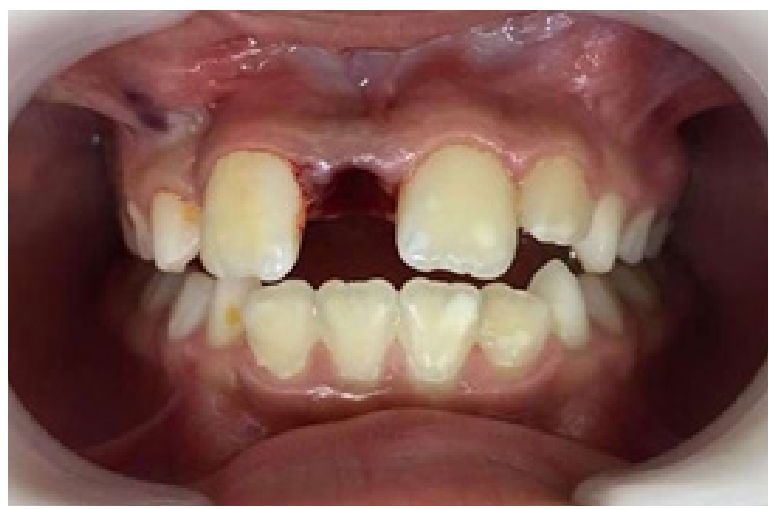

Figura 4. Imagen extra-oral inmediatamente después de la extracción.

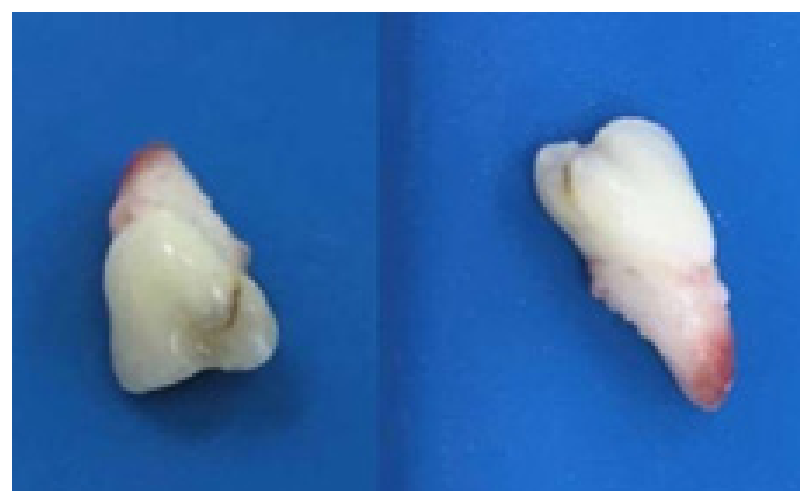

Figura 5. Elemento supernumerario. 
Además, tanto al responsable como al paciente, se le reforzaron las instrucciones sobre la higiene oral y la ausencia de sensibilidad en la región del labio, para que el padre estuviese atento a que el paciente no se mordiera o hiriera el área.

Debido a una pausa en el atendimiento de la clínica integrada del niño y adolescente de la Unigranrio con el fin del semestre lectivo, el paciente fue citado para regresar después de 3 meses. A su regreso, se observó el diastema entre los dientes incisivos centrales superiores con un espacio significativamente menor (Figuras 6 y 7), lo que produjo un cambio en la apariencia y comportamiento del paciente, mostrándose un niño mucho más confiado y cómodo. A pesar de no ser el objeto de estudio del presente artículo, el paciente fue posteriormente referido para ortodoncia, como está indicado en el tratamiento de dientes supernumerarios y diastemas $\operatorname{anteriores}^{23}$ a fin de complementar el tratamiento a través del uso de placa de Hawley con expansor maxilar, buscando la corrección del apiñamiento causado por la falta de espacio en la arcada superior para el posicionamiento del elemento 12 (Figura 8), que se encontraba desplazado palatinamente.

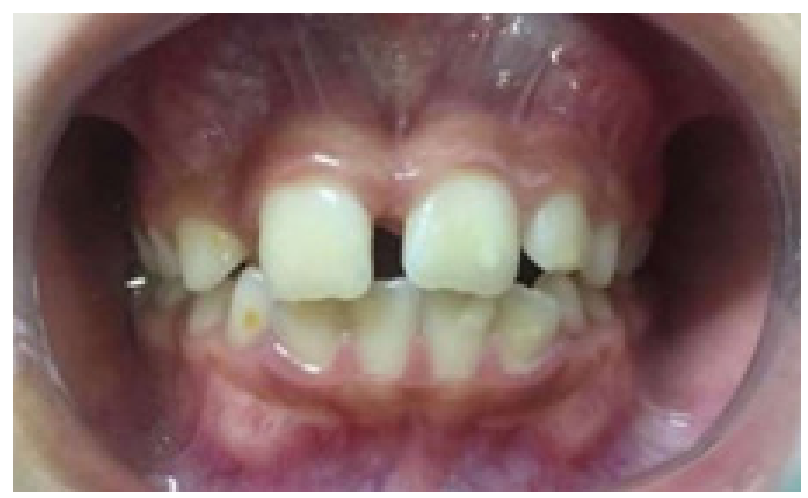

Figura 6. Reevaluación del paciente después de 3 meses con diastema visiblemente menor.

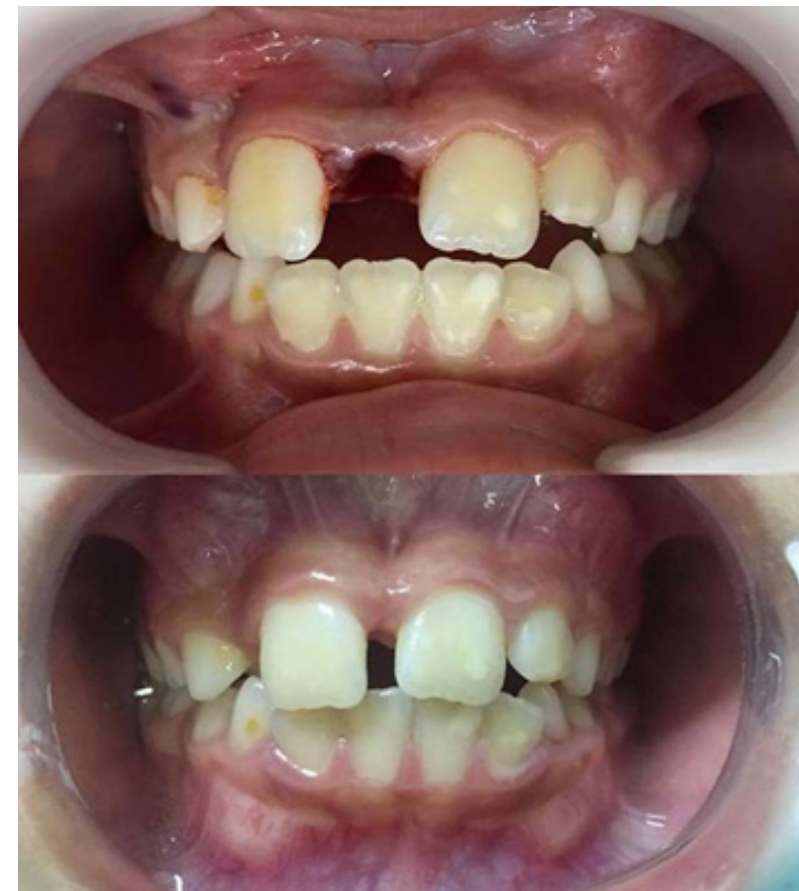

Figura 7. Comparación del momento postquirúrgico inmediato (diastema de $10 \mathrm{~mm}$ ) y reevaluación del paciente a los 3 meses (diastema de apenas $3 \mathrm{~mm}$ ).

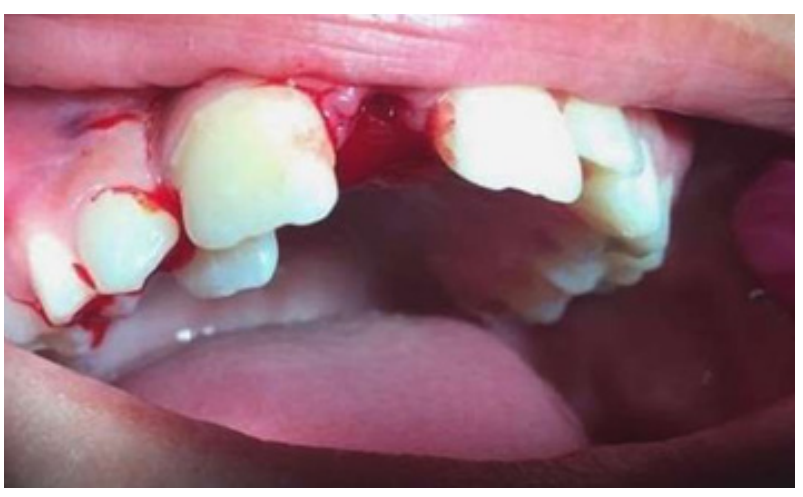

Figura 8. Imagen intra-oral post-extracción, donde se puede ver el elemento 12 desplazado palatinamente.

\section{Discusión}

Como se observó a lo largo del relato del caso, el paciente en estudio desarrolló con el tiempo, características de comportamiento específicas relacionadas 
a la aparición del mesiodens en su dentición primaria, tales como: la postura de retraimiento, timidez, vergüenza al hablar y dificultad de expresarse. Este estudio no pretende dar un punto final al tema, sino presentar una nueva perspectiva relacionada al comportamiento que tienden a presentar los niños portadores de anomalías dentarias durante su infancia, el cual, puede extenderse a su adolescencia e incluso a su vida adulta. ${ }^{10}$

Se sabe que los dientes supernumerarios son una anomalía común, que pueden aparecer tanto en la mandíbula como en el maxilar y ocurren con mayor prevalencia en la dentición permanente que en la dentición primaria. En lo que se refiere al mesiodens, se encuentra con mayor frecuencia en hombres y con forma cónica, como se muestra en el estudio de Jiménez et al. ${ }^{21}$ La mala oclusión generada por el mesiodens puede ser variada, ${ }^{16}$ desde una pequeña desviación de la línea media hasta la posible pérdida de un diente permanente debido a una resorción radicular, generando una extracción precoz. ${ }^{6,12}$ Por lo tanto, las maloclusiones de desarrollo precoz deben ser interceptadas con el objetivo de restituir la oclusión normal lo más rápido posible. ${ }^{22,23}$ En el caso estudiado, la presencia del mesiodens generó un gran desvío de la línea media e impidió que el incisivo lateral superior derecho (12) erupcionara en su posición correcta en la arcada, pues no había espacio suficiente para otro elemento dentario.

Otro aspecto importante se refiere al inicio del establecimiento de la oclusión permanente. Debido a que el mismo se encontraba en una fase de transición y no poseía su dentición permanente completamente formada, el tratamiento fue fundamental para que el espacio ofrecido fuese suficiente para la erupción de los dientes permanentes en el futuro, favoreciendo la formación de una correcta llave de oclusión. ${ }^{7}$

La presencia de una anomalía dentaria en una arcada, puede tener amplia interferencia en la vida de su portador, bien sea en un aspecto estético, funcional o social. Los niños tienden a sufrir más con la aparición de esas anomalías debido al período de desarrollo en que seencuentran. Su personalidad no está completamente formada y otros niños pueden ser crueles en algunas situaciones. ${ }^{6}$

En el caso en estudio, se observó que el mayor compromiso fue presentado en la estética, pues el mesiodens generaba una desarmonía en su sonrisa e incluso durante sus conversaciones, lo que afectó su autoestima y, consecuentemente, se instaló una postura de retraimiento y timidez, como fue observado en el estudio de Varelli et al. ${ }^{6}$ En otro momento, el paciente relató ser llamado "tiburón" por los compañeros de la escuela, lo que ocasionaba alteraciones en su convivencia social, pues decía sentirse excluido.

El responsable del niño relató que el elemento supernumerario había erupcionado junto con los incisivos centrales superiores alrededor de los 7 años. Habría sido fundamental que el diagnóstico y el tratamiento hubiesen sido aplicados precozmente, ${ }^{14}$ para evitar los cambios de comportamiento observados en el paciente, ya que el factor que disminuía su autoestima habría sido eliminado. ${ }^{15}$ Se discute en la literatura que mientras antes se realice el diagnóstico, mejor será el pronóstico del caso. ${ }^{17}$ Para que el diagnóstico precoz pueda ser realizado 
exitosamente, la radiografía panorámica juega un papel fundamental, ya que es la mejor forma rastreo, sin embargo, para casos más complejos en donde la claridad de la línea media se encuentra limitada, tal vez sea necesaria la solicitud de una tomografía computarizada.

El paciente se mostraba como un niño retraído, que sonreía poco para las personas fuera de su convivencia. Una vez realizada la extracción y pasado el tiempo en que regresó a las consultas con un diastema significativamente menor (el espacio que antes era de $10 \mathrm{~mm}$, se presentó con sólo $3 \mathrm{~mm}$ ), se constató que el mismo ya había mejorado la comunicación verbal y la corporal, sonreía para todos y se sentía cómodo al conversar y presentarse, lo que refleja un cambio positivo en su estilo de vida después del tratamiento. La literatura demuestra que el estilo de vida es un componente importante para medir la calidad de vida del paciente, que normalmente seevalúa a través decambios de comportamiento significativos, como los reportados en el presente artículo. Este resultado demuestra que el cambio de comportamiento en las relaciones sociales se hace evidente en el caso de pacientes con anomalías dentarias anteriores tratadas, transformando su manera de ver el mundo y relacionarse con otros.

"Bullying" son prácticas violentas e intencionales realizadas durante la infancia con el objetivo de disminuir o subyugar a otro individuo. En el ambiente escolar puede traer diversos problemas, incluso la dificultad de aprendizaje. Siendo así, la intervención del profesor, de los familiares y orientación de profesionales de la salud en ese ambiente deben priorizar para prevenir este problema. ${ }^{20}$ De acuerdo con Al Bitar, ${ }^{9}$ que realizó un estudio sobre las principales causas del "bullying", en Amán, Jordania, se constató que los dientes eran la principal característica que daba inicio al "bullying". Su prevalencia era del $47 \%$ y predominantemente en el medio masculino. En un estudio realizado en Nigeria, se evidenció que los niños que sufrían de "bullying", les gustaba menos ir a las clases que a los niños que no lo sufrían, lo que podría afectar su atención y desarrollo académico. ${ }^{10}$ Un estudio realizado en niños de 8 a 10 años, concluyó que las características oclusales y el espacio anterior entre los dientes, son características asociadas al empeoramiento en la calidad de vida. ${ }^{18}$ Según Dutra et al. ${ }^{19}$ la mala oclusión, especialmente en dientes anteriores, puede comprometer el bienestar psicosocial del niño.

Poseer esas anomalías puede generar gran sufrimiento psicológico y emocional al paciente. La corrección precoz de estos casos tiende a generar no sólo beneficios estéticos para el niño, sino también la reposición de su autoestima, generando efectos positivos en su desarrollo y evitando complicaciones que puedan surgir en su adolescencia o su vida adulta.

\section{Conclusiones}

Se puede concluir que después de la extracción del mesiodens y con la disminución del diastema anterior, se observaron cambios positivos en el comportamiento del paciente, presentando mejoría en su autoestima e interacciones sociales. 


\section{Referencias bibliográficas}

1. Reis LFG, Allan G, Namba EL, Silva ML, Garcia MA. Dentes supranumerários retidos interferindo no tratamento endodôntico. RSBO 2006; 3(2):20-25.

2. Abreu e Lima F, Motisuki C, Bourdan MM. Mesiodens: detecção e intervenção cirúrgica precoce. RGO. 2002; 50: 69-73.

3. Bezerra PM, Cavalcanti AL. Dentes suprenumerários: revisão de literatura e relato de caso. RevCi med. biol. 2007; 6: 349-356.

4. Shafer WG, Hine MK, Levy BM. Tratado de patologia bucal. 4.ed. Rio de Janeiro: Guanabara Koogan;1987.

5. Alvarez LC, Tavano O. Curso de radiologia em Odontologia. 4.ed. São Paulo, Santos; 1998.

6. Varelli FP, Junior DR, Miranda VC. Correção ortodôntica de pacientes com mesiodens. Ver Uninga. 2012; 8: 6-16.

7. Calneto JO, Cunha DL, Miguel JM. Diastemas interincisais superiores associados a dentes supranumerários-considerações clínicas e relato de caso. J BrasOrtodonOrtop Facial. 2002; 7: 239-44.

8. Mezzomo CS, Tonelotto PR, Mundstock CA. Diagnóstico tardio de mesiodens e sua relação com maloclusão: relato de caso. Rev Fac Odonto Poa.2004; 45: 41-3.

9. Al-bitar Z. et al. Bullying among Jordanian schoolchildren, its effects on school performance, and the contribution of general physical and dentofacial features. Am J Orhod Dentofacial Ortop. 2013; 144:872-8.

10. Chikaodi O, Abdulmanan Y, Emmanuel AT, Muhammad J, Mohammed MA, Izegboya A, Donald $\mathrm{O}$, Balarabe S. Bullying, its effects on attitude towards class attendance and the contribution of physical and dentofacial features among adolescents in Northern Nigeria. Intern Journ of Adlsc Med and Health. 2017 mar 22:1-8.

11. Pedrosa MM. Abordagens terapêuticas para o encerramento de diastemas interincisivos [monografia de mestrado]. Almada (PT): Inst.Sup.de Ciências da Saúde Egas Moniz; 2017.

12. Rocha SC, Vidigal BL, Pereira AC, Fonseca MS, Manzi FR. Etiologia, Diagnóstico e tratamento do mesiodens - relato de caso clínico atípico. Arquivo Brasileiro de Odontologia. 2012; 8: 49-54.

13. Báez-Quintero LC, Percinoto C, Cunha RF, Amaral JG, Hosida TY, Favretto CO, Nagata ME, Delbem ACB. In: CONGRESSO DA FOA UNESP,7., 2017, São Paulo. Mesiodente impactado na linha meia da maxila e assoalho da fossa nasal: caso clínico. São Paulo: Unesp, 2017. 163.

14. Ricci HA, Aranha AMF, Nogueira I, Ferrari JCL, Abreu e Lima FCB. Mesiodens: a importância do diagnóstico precoce. Rev odontol. 2005; 34:

15. Mezzomo CS, Tonelotto PR, Mundstock CA. Diagnóstico tardio de mesiodens e sua relação com a maloclusão: relato de caso. Rev. Fac. Odontol. 2004; 45: 41-3.

16. Cachapuz PF, Siqueira FS, Prietsch JR. Tratamento ortodontico interceptativo de maloclusão causada por mesiodente:relato de caso. Rev. Fac. Odontol. Poa. 2002; 43: 18-22.

17. Marchetti V, Oliveira RV. Mesiodens - dentes supranumerários: diagnóstico, causas e tratamento. Rev. Uninga Review. 2015; 24: 19-23.

18. Sardenberg F, Martins MT, Bendo CB, Pordeus IA, Paiva SM, Auad SM, et al. Malocclusion and oral health-related quality of life in Brazilian school children A population-based study. Angle Orthod. 2013; 83(1): 83-9.

19. Dutra SR, Pretti H, Martins MT, Bendo CB, Vale MP. Impact of malocclusion on the quality of life of children aged 8 to 10 years. Dental Press J Orthod. 2018;23(2):46-53.

20. Cardozo ED, Oliveira APG, Almeida BM, Silva DL, Britto AM, Chevitarese L. A influência do bullying nos aspectos sociais da criança com displasia ectodérmica e o conhecimento de seus responsáveis e professores a respeito da doença: um olhar de enfermagem. Rev. Rede de cuidados em saúde.2016;10:1-14. 
21. Jiménez S, GJ, Medina AC, Crespo O, Tovar R. Manejo clínico de dientes supernumerarios em pacientes pediátricos. Revi Odontoped Latinoam. 2012; 2. 48-58.

22. Kumar A, Shetty RM, Dixit U, Mallikarjun K, Kohli A. Orthodontic Management of Midline Diastema in Mixed Dentition. Int J Clin Pediatr Dent. 2011; 4: 59-63.

23. Sarne O, Shapira Y, Blumer S, Finkelstein T, Schonberger S, Bechor N, Shpack N. Supernumerary Teeth in the Maxillary Anterior Region: The Dilemma of Early Versus Late Surgical Intervention. J Clin Pediatr Dent. 2018; 42: 55-6

Recibido: $17 / 06 / 2018$

Aceptado: 13/03/2019

Correspondencia: Laís Cecilio da Costa, correo: laiscecilio@yahoo.com.br 